\section{Directed Attention}

\section{Ronald Cohen}

Center for Cognitive Aging and Memory, University of Florida, Gainsville, FL, USA

\section{Definition}

Directed attention is the allocation of attention in a "directed" manner to specific information or cognitive processes.

A variety of terms have been used over the years to characterize the varieties of attentional experience, including selective, focused, sustained, divided, split, and directed attention. Some of these terms continue to be widely used to describe elementary component processes underlying attention, most notably selective, focused, and sustained attention, while others like directed and divided attention are best viewed as related to these more fundamental component processes. Directed attention denotes the fact that attention can be focused selectively in a sustained way, though it probably is best thought of as a composite of other more basic forms of attention. Directed attention often implies an "intentional" or overt focusing of attention, in contrast to more covert reactive forms of attention driven by the stimuli that occur. An example of this would be a task in which a subject is instructed to attend to a particular source of information, such as during a dichotic listening paradigm. However, there is considerable variability in how this term is used.

\section{Cross-References}

Attention

Divided Attention

Focused Attention

Selective Attention 